\title{
An Important Announcement from the World Health Organization: "Seconds Save Lives_Clean your Hands": The May 5, 2021, World Health Organization SAVE LIVES: Clean Your Hands campaign
}

\author{
Benedetta Allegranzi ${ }^{1}$, Ermira Tartari ${ }^{1,2,3}$ and Didier Pittet MD, MS $^{3}$ (i) \\ ${ }^{1}$ Infection Prevention and Control Technical and Clinical Hub, Department of Integrated Health Services, World Health Organization, Geneva, Switzerland, \\ ${ }^{2}$ Faculty of Health Sciences, University of Malta, Malta and ${ }^{3}$ Infection Control Programme, University of Geneva Hospitals and Faculty of Medicine, Geneva, \\ Switzerland
}

The year 2020 was unprecedented in many ways, one of which was the tremendous attention given to appropriate hand hygiene practices in the fight against severe acute respiratory coronavirus virus 2 (SARS-CoV-2). Hand hygiene has finally gained global recognition from policy makers, health managers, healthcare workers, and the general public as a keystone in infection prevention. The World Health Organization (WHO) placed increased focus on hand hygiene in the context of the global coronavirus disease 2019 (COVID-19) pandemic, in addition to its longstanding efforts through both the global SAVE LIVES: Clean Your Hands campaign and the Water, Sanitation and Hygiene (WASH) Programme.

In the context of the pandemic, the WHO launched several initiatives, including the new WHO and UNICEF Hand Hygiene for All initiative (https://www.who.int/water_sanitation_health/ sanitation-waste/sanitation/hand-hygiene-for-all/en/) to consistently improve hand hygiene practices as a whole-of-society approach to stop the spread of SARS-CoV-2 and sustain good practices beyond the pandemic. To achieve these goals, adequate infrastructures should be provided in healthcare and public settings, including schools and public transportation, and appropriate behavior to clean hands when needed should be undertaken by all key players.

This year, the WHO's SAVE LIVES: Clean Your Hands campaign focuses on achieving appropriate hand hygiene action at the point of care. This campaign has been at the core of WHO patient safety strategies during healthcare delivery for many years, but it is now more critical than ever. Furthermore, 2021 has been designated the International Year of Health and Care Workers (https://www.who.int/campaigns/annual-theme/year-of-healthand-care-workers-2021); focusing on their protection is also paramount.

The first prerequisite for effective implementation of hand hygiene action at the point of care is "system change," meaning that the appropriate infrastructure and supplies should be available at the point of care so that health workers can clean their hands promptly when needed. To do so, requires reliable and uninterrupted provision of good-quality alcohol-based hand rub

Author for correspondence: Didier Pittet, E-mail: Didier.Pittet@hcuge.ch

Cite this article: Allegranzi B, Tartari E, and Pittet D. (2021). An Important Announcement from the World Health Organization: "Seconds Save Lives-Clean your Hands": The May 5, 2021, World Health Organization SAVE LIVES: Clean Your Hands campaign. Infection Control \& Hospital Epidemiology, 47: 649-651, https:// doi.org/10.1017/ice.2021.93
(ABHR)are required, as well as supplies of clean water, soap, single-use towels and an adequate number of functioning sinks. Although effective infection prevention and control (IPC) programs in healthcare facilities should meet the WHO minimum requirements, ${ }^{1}$ the 2020 global WASH report revealed that 1 in 3 facilities do not have adequate hand hygiene stations at the point of care. ${ }^{2}$ A recent systematic review showed that hand hygiene compliance is only ${ }^{\sim} 9 \%$ during care of critically ill patients in low-income countries. ${ }^{3}$ Such shocking data, in conjunction with the ongoing COVID-19 pandemic, highlight the urgent need for additional efforts to strengthen global compliance and champion best practices. Although vaccines are starting to be delivered, hand hygiene and appropriate use of personal protective equipment remain crucial for safe care of both COVID-19 and non-COVID-19 patients.

Effective hand hygiene not only reduces the burden of healthcare-associated infections and the spread of antimicrobial resistance, but it is also a key IPC measure for safe COVID-19 vaccination. ${ }^{4} \mathrm{ABHR}$ is the preferred method for hand hygiene in health care because it can be easily accessible at the point of care, kills microorganisms quickly (within 20-30 seconds), and is well tolerated by the skin. These advantages can help overcome behavioral barriers to compliance. In the light of current shortages, many countries have successfully established local ABHR production as a low-cost alternative within facilities, using WHO-recommended formulations. ${ }^{5}$

To highlight the urgent need to save lives by implementing best practices in healthcare delivery, the slogan for May 5, 2021, is "Seconds Save Lives-Clean your Hands" (Fig. 1). The WHO campaign calls to action key stakeholders (Table 1) who can play critical roles in achieving optimal hand hygiene at the point of care, in both the current situation and a broader sense, helping to strengthen society involvement as promoted by the Hand Hygiene for All initiative.

\footnotetext{
Acknowledgments. The authors alone are responsible for the views expressed in this article; it does not necessarily represent the views, decisions, or policies of the institutions with which they are affiliated.
}

Financial support. Support for this article was provided by the World Health Organization (WHO), Geneva, Switzerland, and the Infection Control Program, University of Geneva Hospitals and Faculty of Medicine, Geneva, Switzerland. Hand hygiene research activities at the SPCI/WCC are also supported by the Swiss National Science Foundation (grant no. 32003B_163262).

Conflicts of interest. All authors report no conflicts of interest relevant to this article. 


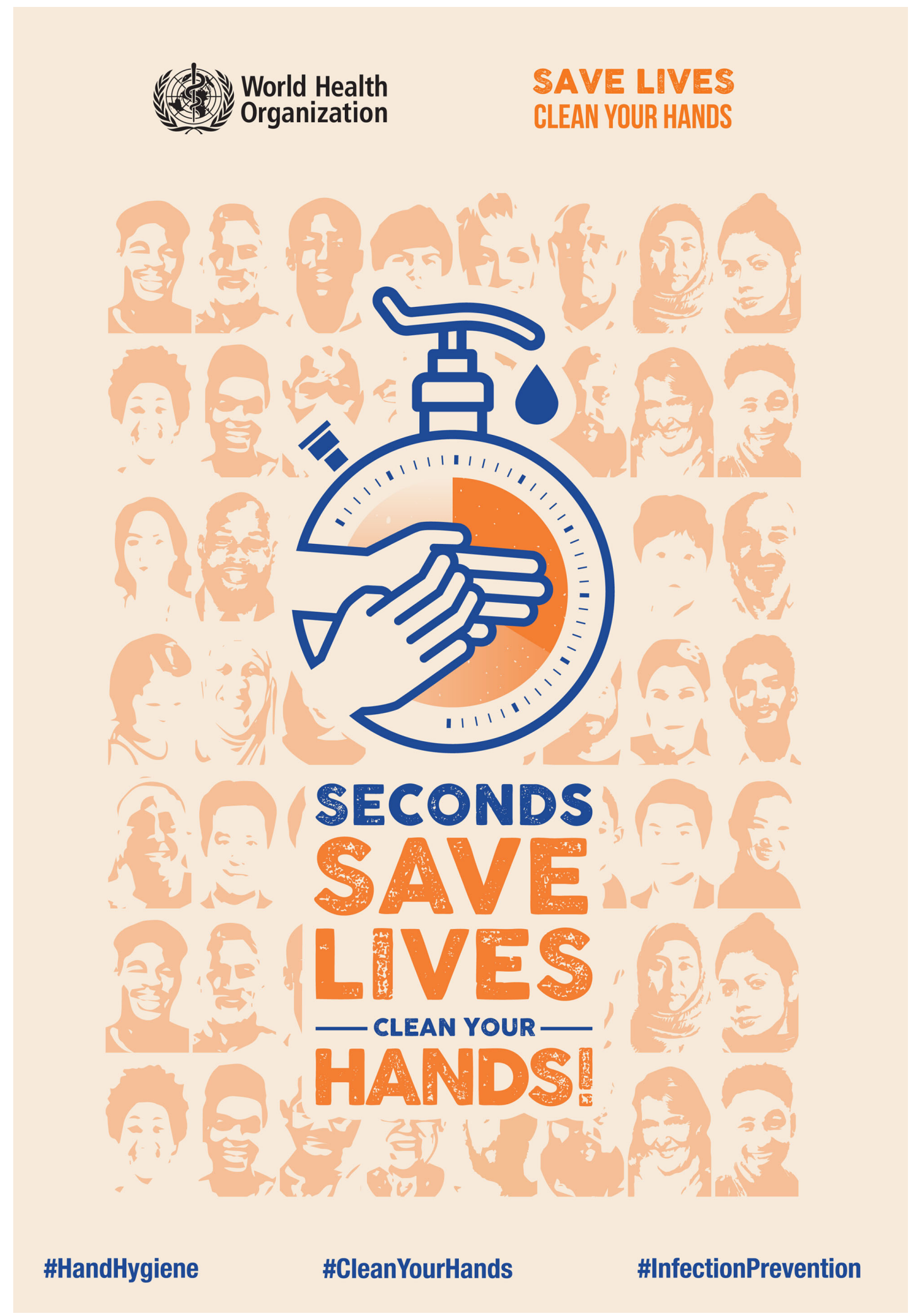

Fig. 1. May 5, 2021, "Seconds save lives—Clean your hands" campaign slogan and main promotional image (2021 hashtags: \#HandHygiene, \#CleanYourHands, \#lnfectionPrevention). 
Table 1. May 5, 2021, WHO SAVE LIVES: Clean Your Hands Campaign Calls to Action ${ }^{\text {a }}$

\begin{tabular}{lc}
\hline Campaign Participants & Call to Action \\
\hline Healthcare workers & "Now more than ever, clean your hands at the point of care."b \\
\hline IPC practitioners & "Champion and mentor clean hands at the point of care." \\
\hline Facility managers & "Ensure hand hygiene supplies are available at every point of care." \\
\hline Policy makers & "Invest now to ensure hand hygiene for all." \\
\hline Patients and families & "Help us to help you: please clean your hands." \\
\hline Vaccinators & "Clean your hands with every vaccine." \\
\hline General public & "Make clean hands your habit - it protects us all." \\
\hline
\end{tabular}

Note. IPC, infection prevention and control.

${ }^{a}$ All the information is available on the WHO SAVE LIVES: Clean Your Hands campaign webpage (https://www.who.int/campaigns/save-lives-clean-your-hands), including an advocacy tool kit offering guidance on the campaign's objectives, key messages and how to get involved.

bPoint of care refers to the to the place where 3 elements come together: the patient, the healthcare worker, and care or treatment involving contact with the patient or their surroundings (as published in the WHO guidelines on hand hygiene in health care).

\section{References}

1. Minimum requirements for infection prevention and control programmes. World Health Organization website. https://apps.who.int/iris/handle/ 10665/330080. Published 2019. Accessed March 10, 2021.

2. Global progress report on WASH in health care facilities: fundamentals first. World Health Organization website. https://www.who.int/publications/i/ item/9789240017542. Published 2020. Accessed March 10, 2021.

3. Lambe KA, Lydon S, Madden C, et al. Hand hygiene compliance in the ICU: a systematic review. Critical Care Med 2019;47:1251-1257.
4. Aide memoire: infection prevention and control (IPC) principles and procedures for COVID-19 vaccination activities. Geneva: World Health Organization; 2021 (https://www.who.int/publications/i/item/ who-2019-ncov-vaccination-IPC-2021-1). Published 2021. Accessed March 10, 2021.

5. Guide to local production: WHO-recommended handrub formulations. Geneva: World Health Organization; 2010 (https://www.who.int/publications/ i/item/WHO-IER-PSP-2010.5) 